\title{
Phase-Shift Cyclic-Delay Diversity for MIMO OFDM Systems
}

\author{
Young-Han Nam, Lingjia Liu, and Jianzhong (Charlie) Zhang \\ Wireless Solutions Lab, Samsung Telecommunications America, Richardson, TX 75082, USA \\ Correspondence should be addressed to Young-Han Nam, ynam@sta.samsung.com
}

Received 1 September 2009; Accepted 29 November 2009

Academic Editor: Hongxiang Li

Copyright ( 2010 Young-Han Nam et al. This is an open access article distributed under the Creative Commons Attribution License, which permits unrestricted use, distribution, and reproduction in any medium, provided the original work is properly cited.

Phase-shift cyclic-delay diversity (PS CDD) scheme and space-frequency-block-code (SFBC) PS CDD are developed for multipleinput-multiple-output (MIMO) orthogonal frequency division multiplexing (OFDM) systems. The proposed PS CDD scheme preserves the diversity advantage of traditional CDD in uncorrelated multiantenna channels, and furthermore removes frequencyselective nulling problem of the traditional CDD in correlated multiantenna channels.

\section{Introduction}

It is well known that a multiple-input-multiple-output (MIMO) transmission system can provide benefits on throughput and reliability in multipath fading channels over single-antenna systems [1]. These benefits are now being realized; MIMO transmission schemes have been adopted in most wireless standards including 3GPP long-term evolution (LTE), 3GPP LTE-Advanced, WiMax, and IEEE $802.16 \mathrm{~m}$, where these standards are based on orthogonal frequency division multiple access (OFDMA). OFDMA became popular partly because it is sum-rate optimal for general single-input-single-output (SISO) channels, and the probability of OFDMA being optimal is nonnegligible for MIMO channels [2].

In particular, transmit diversity $(\mathrm{TxD})$ schemes are utilized to realize the reliability benefits of multi-antenna systems in slow-fading environment without channel state information (CSI) available at the transmitter side, by providing multiple signals conveying the same information over different spatial channels. In OFDM-based systems, Alamouti space-frequency block code (SFBC) [3] and cyclic delay diversity (CDD) [4] are widely adopted TxD schemes for 2-transmit antenna diversity $(2-\mathrm{TxD})$ systems. The SFBC scheme has advantages over the other $\mathrm{TxD}$ shemes; it is simple to encode at the transmitter side and easy to decode at the receiver side while still achieving the optimal uncoded diversity gain in $2 \times 1$ Rayleigh fading channels (i.e., with two transmit and one receive antennas). On the other hand,
CDD with small delay is an attractive diversity scheme in OFDM systems, in a sense that it requires only one set of pilot signals, as opposed to the SFBC scheme where two sets of pilot signals are required. However, CDD has some drawbacks such as that it does not have uncoded diversity and thus the block-error rate (BLER) performance is worse than the uncoded diversity schemes like SFBC, and that it has frequency-selective nulls in antenna-correlated channels.

In this paper, we develop and analyze a new $\mathrm{TxD}$ scheme for OFDM systems, phase-shift CDD (PS CDD), which takes advantages from both SFBC and CDD, and at the same time mitigates the issue of frequency-selective nulls. The performance of the introduced transmit diversity scheme is evaluated through numerical simualtion results.

\section{System Model}

We consider a MIMO OFDM system, with $M$ transmit antennas and $N$ receive antennas, where $M=1,2, \ldots$ and $N=1,2, \ldots$. For each subcarrier $k=0,1, \ldots, N_{\mathrm{FFT}}-1$, where $N_{\text {FFT }}$ is the FFT size for the OFDM system, a received signal is described as in the following equation:

$$
\mathbf{y}_{k}=\mathbf{H}_{k} \mathbf{x}_{k}+\mathbf{w}_{k}
$$

where $\mathbf{y}_{k} \in C^{N \times 1}$ is a received vector, $\mathbf{H}_{k} \in C^{N \times M}$ is a MIMO channel matrix, $\mathbf{x}_{k} \in C^{M \times 1}$ is a transmitted vector, and $\mathbf{w}_{k} \in$ $C^{N \times 1}$ is an additive white Gaussian noise vector with mean 0 , covariance matrix $\operatorname{diag}\left(\sigma^{2}\right)$. Here, $C$ is the set of complex numbers. 
In this paper, we focus on $\mathrm{TxD}$ schemes used for robust transmissions in various channel conditions, such as highDoppler channels and highly frequency-selective channels. Such TxD schemes transmit only one channel-coded stream to ensure maximum reliability, while sacrificing spectral efficiency. We note that general TxD schemes may transmit multiple streams [1] and can also be used for multi-user setting [5] when spatial degrees of freedom of a MIMO channel are greater than one. Among this class of TxD schemes, Alamouti SFBC and CDD are two popular TxD schemes in OFDM-based wireless transmission systems.

\section{Background: 2-TxD Schemes}

Alamouti SFBC can be described in a 2-transmit and 1receive antenna system. For an SFBC transmission, two transmit signals at two adjacent subcarriers are paired, which we denote as $\mathbf{x}_{k}$ and $\mathbf{x}_{k+1}$. These two vectors are constructed in such a way that

$$
\mathbf{x}_{k}=\sqrt{\frac{P}{2}}\left[\begin{array}{c}
s_{k} \\
s_{k+1}
\end{array}\right], \quad \mathbf{x}_{k+1}=\sqrt{\frac{P}{2}}\left[\begin{array}{c}
-s_{k+1}^{*} \\
s_{k}^{*}
\end{array}\right],
$$

where $s_{k}$ and $s_{k+1}$ are modulated symbols with variance 1 and $P$ is total transmit power at each subcarrier. Under this construction, we are able to obtain an orthogonal system representation at the receiver side, that is, the system transfer matrix is orthogonal:

$$
\left[\begin{array}{c}
y_{k} \\
y_{k+1}^{*}
\end{array}\right]=\sqrt{\frac{P}{2}}\left[\begin{array}{cc}
h_{11} & h_{12} \\
h_{12}^{*} & -h_{11}^{*}
\end{array}\right]\left[\begin{array}{c}
s_{k} \\
s_{k+1}
\end{array}\right]+\left[\begin{array}{c}
w_{k} \\
w_{k+1}^{*}
\end{array}\right],
$$

where $h_{11}$ and $h_{12}$ are the channel coefficients between the receive antenna and each of the transmit antennas at subcarriers $k$ and $k+1$, with an assumption that the channel does not vary in the two subcarriers. Due to the orthogonal system transfer matrix, Alamouti SFBC is called an orthogonal TXD scheme. Utilizing the orthogonality property, we can detect $s_{k}$ and $s_{k+1}$, from the two Alamouti receiver equations as follows:

$$
\begin{aligned}
& h_{11}^{*} y_{k}+h_{12} y_{k+1}^{*}=\sqrt{\frac{P}{2}}\left(\left|h_{11}\right|^{2}+\left|h_{12}\right|^{2}\right) s_{k}+h_{11}^{*} w_{k}+h_{12} w_{k+1}^{*}, \\
& h_{12}^{*} y_{k}-h_{11} y_{k+1}^{*}=\sqrt{\frac{P}{2}}\left(\left|h_{11}\right|^{2}+\left|h_{12}\right|^{2}\right) s_{k+1}+h_{12}^{*} w_{k}-h_{11} w_{k+1}^{*} .
\end{aligned}
$$

From the receiver equations, one can easily verify that the received signal-to-noise ratios (SNRs) of the two modulated symbols are the same and equal to $\left(\left|h_{11}\right|^{2}+\left|h_{12}\right|^{2}\right) P /\left(2 \sigma^{2}\right)$, which reveals the uncoded diversity gain of the SFBC scheme.

When the number of receive antennas is greater that 1 , maximal ratio combining (MRC) would produce a received SNR of

$$
\sum_{n}\left(\left|h_{n 1}\right|^{2}+\left|h_{n 2}\right|^{2}\right) P /\left(2 \sigma^{2}\right)
$$

where $h_{n 1}$ and $h_{n 2}$ are the channel coefficients between receive antenna $n$ and each of the transmit antennas at subcarriers $k$ and $k+1$. Note that when the number of transmit antennas is larger than 2, no orthogonal $\mathrm{TxD}$ schemes have been found achieving the full rate [6]. One popular extension of SFBC for 4-Tx antenna transmitter is frequency-switched transmit diversity (FSTD) [4]. For an SFBC-FSTD transmission, four adjacent subcarriers, $k, k+1$, $k+2$, and $k+3$ are grouped. On the first two subcarriers, that is, $k$ and $k+1$, one SFBC pair is transmitted on the first and the second antennas, while the third and the fourth antennas are turned off. On the third and the fourth subcarriers, that is, $k+2$ and $k+3$, another SFBC pair is transmitted on the third and the fourth antennas, while the first and the second antennas are turned off. SFBC-FSTD is easy to code and decode since it keeps the orthogonality property and achieves coded diversity across four transmit antennas. However, we need four pilot signals for demodulation of SFBC-FSTD, which may increase pilot overhead of a system.

On the other hand, CDD is a coded TxD scheme in an OFDM system, which can be designed for arbitrary number of Tx antennas. In two-transmit and one-receive antenna system, at subcarrier $k$, a transmit signal $\mathbf{x}_{k}$ coded with CDD is

$$
\mathbf{x}_{k}=\sqrt{\frac{P}{2}}\left[\begin{array}{c}
s_{k} \\
e^{j k \delta} s_{k}
\end{array}\right]
$$

where $\delta$ is a positive number called CDD delay (e.g., $\delta=$ $\left.2 \pi / N_{\mathrm{FFT}}\right)$. Then, a received signal at subcarrier $k$ is written as

$$
y_{k}=\left[\begin{array}{ll}
h_{11} & h_{12}
\end{array}\right] \sqrt{\frac{P}{2}}\left[\begin{array}{c}
s_{k} \\
e^{j k \delta} s_{k}
\end{array}\right]+w_{k}=\sqrt{\frac{P}{2}}\left(h_{11}+h_{12} e^{j k \delta}\right) s_{k}+w_{k} .
$$

As we can see from the receiver equation, CDD does not give uncoded diversity, as received SNR is $\left|h_{11}+h_{12} e^{j k \delta}\right|^{2} P /\left(2 \sigma^{2}\right)$. However, CDD in combination of channel coding across modulation symbols $\left\{s_{k}\right\}$ mapping to multiple subcarriers increases a coded diversity gain, as CDD increases the frequency selectivity of the composite channel: $h_{11}+h_{12} e^{j k \delta}$. We also note that with $\mathrm{CDD}$, a receiver needs to know only the composite channel $h_{11}+h_{12} e^{j k \delta}$ for demodulation, especially when the delay $\delta$ is small so that the channel does not vary abruptly over subcarriers. This is one benefit of CDD over SFBC which requires knowledge of two channels.

CDD can be easily extended to cases where the number of Tx antennas is grater than 2. For example, when the number of Tx antennas is 4 , we have a transmit signal $\mathbf{x}_{k}$ coded with CDD at subcarrier $k$ as

$$
\mathbf{x}_{k}=\sqrt{\frac{P}{4}}\left[\begin{array}{c}
s_{k} \\
e^{j k \delta} s_{k} \\
e^{j 2 k \delta} s_{k} \\
e^{j 3 k \delta} s_{k}
\end{array}\right] .
$$


A well-known drawback of CDD is frequency-selective nulling. As CDD artificially increases frequency selectivity, in some subcarriers, the two component channels of the composit channel, $h_{11}+h_{12} e^{j k \delta}$, coherently add, while in some other subcarriers, they destructively add. This problem becomes severer when the two channels $h_{11}$ and $h_{12}$ are correlated, which occurs when the two transmit antennas are geometrically close. This particular issue of CDD prevented it from being accepted as robust $\mathrm{TxD}$ schemes in wireless communication implementations, despite the benefits of CDD.

Reviewing these two TxD schemes of SFBC and CDD, in summary, we realize that SFBC is robust but not extendable to systems with large number of transmit antennas, while CDD is easily extendable and requires only one pilot signal but not robust in correlated channels. In the sequel, we develop new TxD schemes taking the advantages of both schemes while still ensuring robustness in correlated channels.

\section{Design of 4-Tx Diversity Schemes}

4.1. Phase-Shift Cyclic Delay Diversity (PS CDD). We recall that a major problem of CDD is nonrobustness in correlated channels when two terms from $h_{11}+h_{12} e^{j k \delta}$ destructively add. When the number of Tx antennas is greater than or equal to 4 , a simple variation of CDD may prevent frequency nulling from occuring. Instead of giving the same phase component on the signals transmitted in the four transmit antennas as in (8), we attempt to apply a phase shift of $\phi$ in the signal transmitted in the fourth antenna. With this phase shift CDD (PS CDD), transmit signal $\mathbf{x}_{k}$ coded at subcarrier $k$ is

$$
\mathbf{x}_{k}=\sqrt{\frac{P}{4}}\left[\begin{array}{c}
s_{k} \\
e^{j k \delta} s_{k} \\
e^{j 2 k \delta} s_{k} \\
e^{j(3 k \delta+\phi)} s_{k}
\end{array}\right] .
$$

In thise case, the composite channel at the receiver's point of view is $h_{11}+h_{12} e^{j k \delta}+h_{13} e^{2 j k \delta}+h_{14} e^{j(3 k \delta+\phi)}$.

To facilitate the analysis of performance of (9) in strongly correlated channels, we assume

$$
h_{11}=h_{12}=h_{13}=h_{14}=h .
$$

Accordingly, the composite channel becomes

$$
h\left(1+e^{j k \delta}+e^{j 2 k \delta}+e^{j(3 k \delta+\phi)}\right) .
$$

To gain some insights on this approach, let $\delta=2 \pi / N_{\mathrm{FFT}}$, $\phi=\pi$, and $N_{\mathrm{FFT}}=1024$ and compare the composite channel powers of CDD scheme (8) and PS CDD (9) normalized by $|h|^{2}$, as shown in Figure 1. In the figure, we can see that CDD suffers from frequency-selective nulls at $N_{\mathrm{FFT}} / 4, N_{\mathrm{FFT}} / 2$ and $3 N_{\mathrm{FFT}} / 4$, while PS CDD does not have frequency nulls. Recalling that CDD intentionally introduces frequency selectivity for additional frequency diversity, we want to have PS CDD that has a property of having good

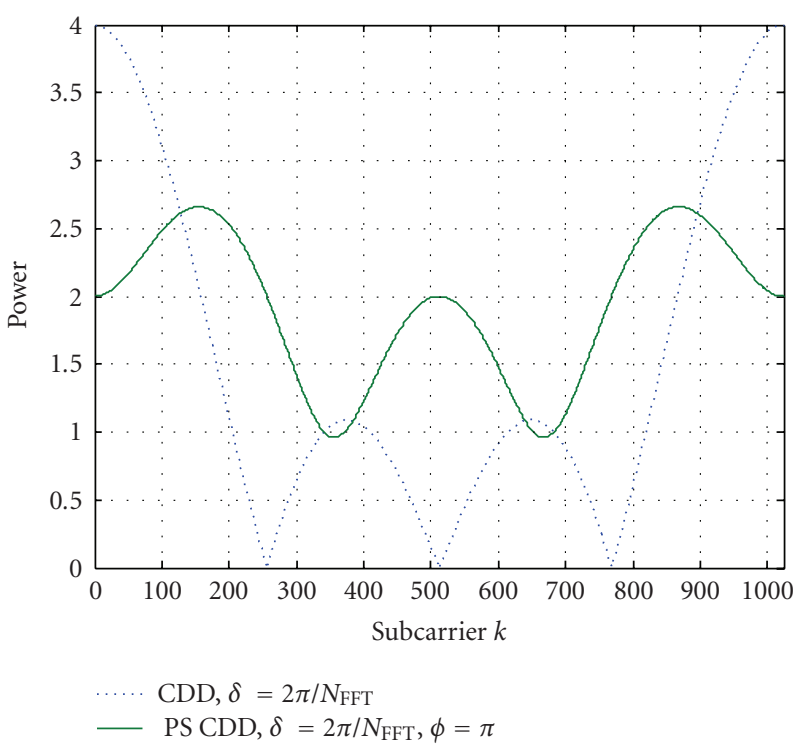

Figure 1: Comparison of powers of composite channels with CDD and PS CDD.

frequency selectivity, while not suffering from frequency nulls. We may characterize this goal by considering the following objective function:

$$
\underset{\phi}{\operatorname{maximize}}\left(P_{\max }-P_{\min }\right) P_{\min },
$$

where $P_{\min }$ and $P_{\max }$ are the minimum power and the maximum power, respectively, of a scheme across all the subcarriers. We find that $\left(P_{\max }-P_{\min }\right) P_{\min }$ of the CDD and the PS CDD in Figure 1 are 0 and 1.6323, and hence under this objective function, PS CDD is better than CDD, in terms of both introducing frequency selectivity and keeping minimum power large. We note that the optimal $\phi$ with (9) can be found with numerical method.

The most general form of PS CDD can be written as

$$
\mathbf{x}_{k}=\sqrt{\frac{P}{4}}\left[\begin{array}{c}
s_{k} \\
e^{j\left(k \delta_{1}+\phi_{1}\right)} s_{k} \\
e^{j\left(k \delta_{2}+\phi_{2}\right)} s_{k} \\
e^{j\left(k \delta_{3}+\phi_{3}\right)} s_{k}
\end{array}\right],
$$

where we may optimize the performance with choosing parameters $\delta$ 's and $\phi$ 's. We also note that for demodulation PS CDD signal, we need only one pilot signal for the composite channel $h_{11}+h_{12} e^{j\left(k \delta_{1}+\phi_{1}\right)}+h_{13} e^{j\left(k \delta_{2}+\phi_{2}\right)}+h_{14} e^{j\left(k \delta_{3}+\phi_{3}\right)}$.

\subsection{Space-Frequency Block-Code with Phase-Shift Cyclic Delay} Diversity (SFBC PS CDD). In Section 4.1, we have introduced PS CDD that does not suffer from frequency-selective nulls, while keeping the coded diversity benefit of CDD and maintaining the required number of pilot signals to be one. In this section, we combine Alamouti SFBC and PS CDD, so that a new TxD scheme can enjoy uncoded diversity while keeping some benefits of PS CDD. 


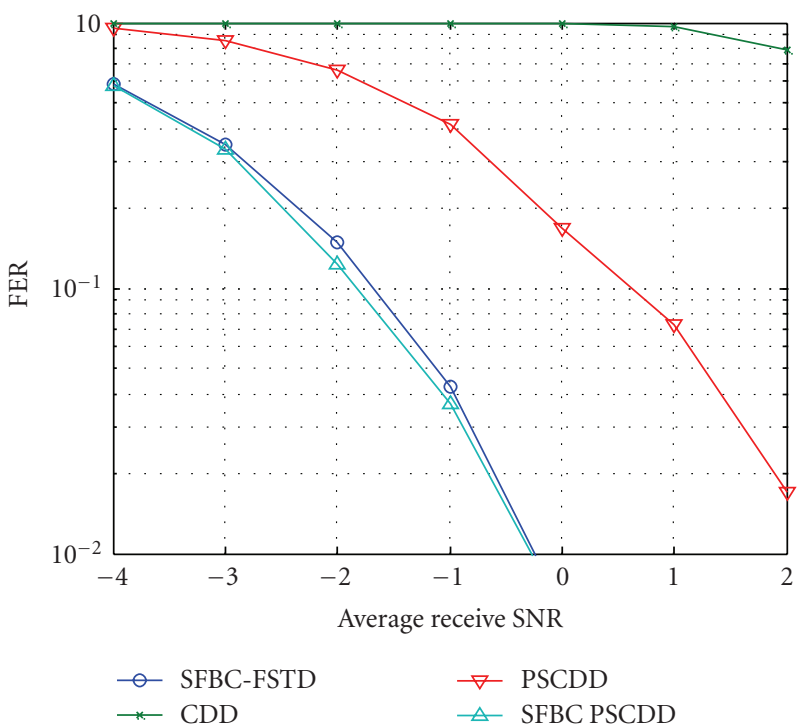

FIGURE 2: BLER performance in correlated channels.

For an SFBC PS CDD transmission, two transmit signals at two adjacent subcarriers are paired, which we denote $\mathbf{x}_{k}$ and $\mathbf{x}_{k+1}$. These two vectors are constructed in such a way that

$\mathbf{x}_{k}=\sqrt{\frac{P}{4}}\left[\begin{array}{c}s_{k} \\ e^{j\left(k \delta_{1}+\phi_{1}\right)} s_{k+1} \\ e^{j\left(k \delta_{2}+\phi_{2}\right)} s_{k} \\ e^{j\left(k \delta_{3}+\phi_{3}\right)} s_{k+1}\end{array}\right], \quad \mathbf{x}_{k+1}=\sqrt{\frac{P}{4}}\left[\begin{array}{c}-s_{k+1}^{*} \\ e^{j\left(k \delta_{1}+\phi_{1}\right)} s_{k}^{*} \\ -e^{j\left(k \delta_{2}+\phi_{2}\right)} s_{k+1}^{*} \\ e^{j\left(k \delta_{3}+\phi_{3}\right)} s_{k}^{*}\end{array}\right]$.

With this construction, we obtain an orthogonal system of equations at the receiver side:

$$
\left[\begin{array}{c}
y_{k} \\
y_{k+1}^{*}
\end{array}\right]=\sqrt{\frac{P}{2}}\left[\begin{array}{cc}
\tilde{h}_{11} & \tilde{h}_{12} \\
\tilde{h}_{12}^{*} & -\tilde{h}_{11}^{*}
\end{array}\right]\left[\begin{array}{c}
s_{k} \\
s_{k+1}
\end{array}\right]+\left[\begin{array}{c}
w_{k} \\
w_{k+1}^{*}
\end{array}\right],
$$

where

$$
\begin{aligned}
& \tilde{h}_{11}=\left(h_{11}+e^{j\left(k \delta_{2}+\phi_{2}\right)} h_{13}\right), \\
& \tilde{h}_{12}=\left(e^{j\left(k \delta_{1}+\phi_{1}\right)} h_{12}+e^{j\left(k \delta_{3}+\phi_{3}\right)} h_{14}\right) .
\end{aligned}
$$

From (14), (15), and (16), we see that SFBC PS CDD construction reduces to a 2 -Tx SFBC scheme, which ensures easy decodability relying on orthogonal structure and allows us to have uncoded diversity. Furthermore, each of the effective channels $\tilde{h}_{11}$ and $\tilde{h}_{12}$ is constructed with CDD, which allows us to have coded diversity with intentionally introduced frequency selectivity and facilitates demodulation of SFBC PS CDD using two pilot signals for $\tilde{h}_{11}$ and $\tilde{h}_{12}$.

\section{Numerical Results}

In this section, we present numerical simulation results of block-error rate (BLER, or frame error rate, FER)

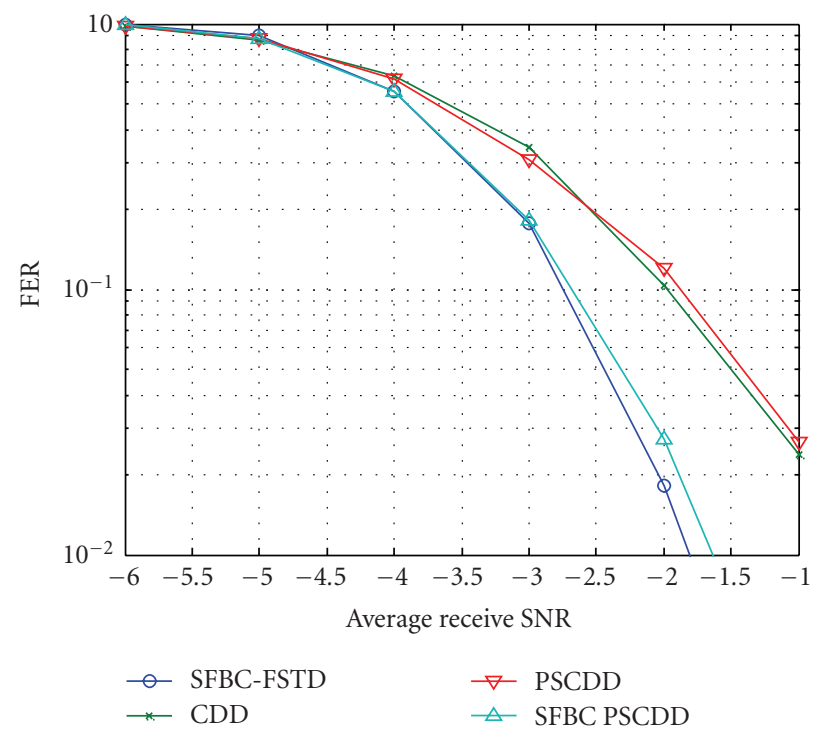

FIGURE 3: BLER performance in uncorrelated channels.

performance comparing the $\mathrm{TxD}$ schemes introduced in this paper and some existing schemes such as CDD and SFBCFSTD. For the simulation, ITU typical urban 6-path channel model (TU-6) has been used and $120 \mathrm{~km} / \mathrm{hr}$ is assumed for terminal speed. Furthermore, we consider a MIMO channel with 4-transmit and 2-receive antennas, where 4 transmit antennas are correlated with correlation coefficients 0.9 and 0 , while 2 receive antennas are uncorrelated. For channel coding, 3GPP Turbo code [7] is used with code rate $1 / 3$, and QPSK modulation is used. Channel-coded and modulated signals go through 6 distributed sets of 12 subcarriers in each time slot (or per block of time). For demodulation, perfect (or ideal) channel estimation is assumed. At the receiver, maximal ratio combing is used followed by perreceive-antenna SFBC decoder.

Figure 2 shows BLER curves obtained with various $\mathrm{TxD}$ schemes under highly correlated channels whose correlation coefficient is 0.9. As we discussed earlier, CDD performs worse than the others. As PS CDD removes frequency nulls, the performance is better than CDD. Both SFBC-FSTD and SFBC-PSCDD perform the best among these four schemes. Considering the fact that SFBC-PSCDD requires only two pilot signals, SFBC-PSCDD can potentially achieve larger spectral efficiency than SFBC-FSTD. On the other hand, Figure 3 shows the BLER curves obtained under uncorrelated channels. In the uncorrelated case, SFBC-FSTD and SFBCPSCDD show similar performance and outperform PSCDD and CDD.

\section{Conclusion}

In this paper, we have introduced phase-shift cyclic delay diversity PS CDD and SFBC PS CDD schemes. The proposed schemes treat frequency-selective nulling problem of traditional CDD. In particular, SFBC PS CDD takes benefits of both SFBC and PS CDD, and achieves robust block-error 
rate performance in both highly correlated and uncorrelated channels, while requiring only two pilot signals, as opposed to the well-known SFBC-FSTD requiring four pilot signals.

\section{References}

[1] L. Zheng and D. N. C. Tse, "Diversity and multiplexing: a fundamental tradeoff in multiple-antenna channels," IEEE Transactions on Information Theory, vol. 49, no. 5, pp. 10731096, 2003.

[2] H. Li and H. Liu, "On the optimality of OFDMA MIMO channels," in Proceedings of the 40th Asilomar Conference on Signals, Systems, and Computers (ACSSC '06), pp. 1757-1761, Pacific Grove, Calif, USA, October 2006.

[3] S. M. Alamouti, "A simple transmit diversity technique for wireless communications," IEEE Journal on Selected Areas in Communications, vol. 16, no. 8, pp. 1451-1458, 1998.

[4] F. Khan, LTE for 4G Mobile Broadband, Cambridge University Press, Cambridge, UK, 1st edition, 2009.

[5] Y.-H. Nam and H. E. Gamal, "On the optimality of lattice coding and decoding in multiple access channels," in Proceedings of the IEEE International Symposium on Information Theory (ISIT '07), pp. 211-215, Nice, France, 2007.

[6] V. Tarokh, H. Jafarkhani, and A. R. Calderbank, "Space-time block codes from orthogonal designs," IEEE Transactions on Information Theory, vol. 45, no. 5, pp. 1456-1467, 1999.

[7] 3GPP TS 36.212 ver 8.7.0, "Evolved Universal Terrestrial Radio Access (E-UTRA); Multiplexing and channel coding," June 2009. 

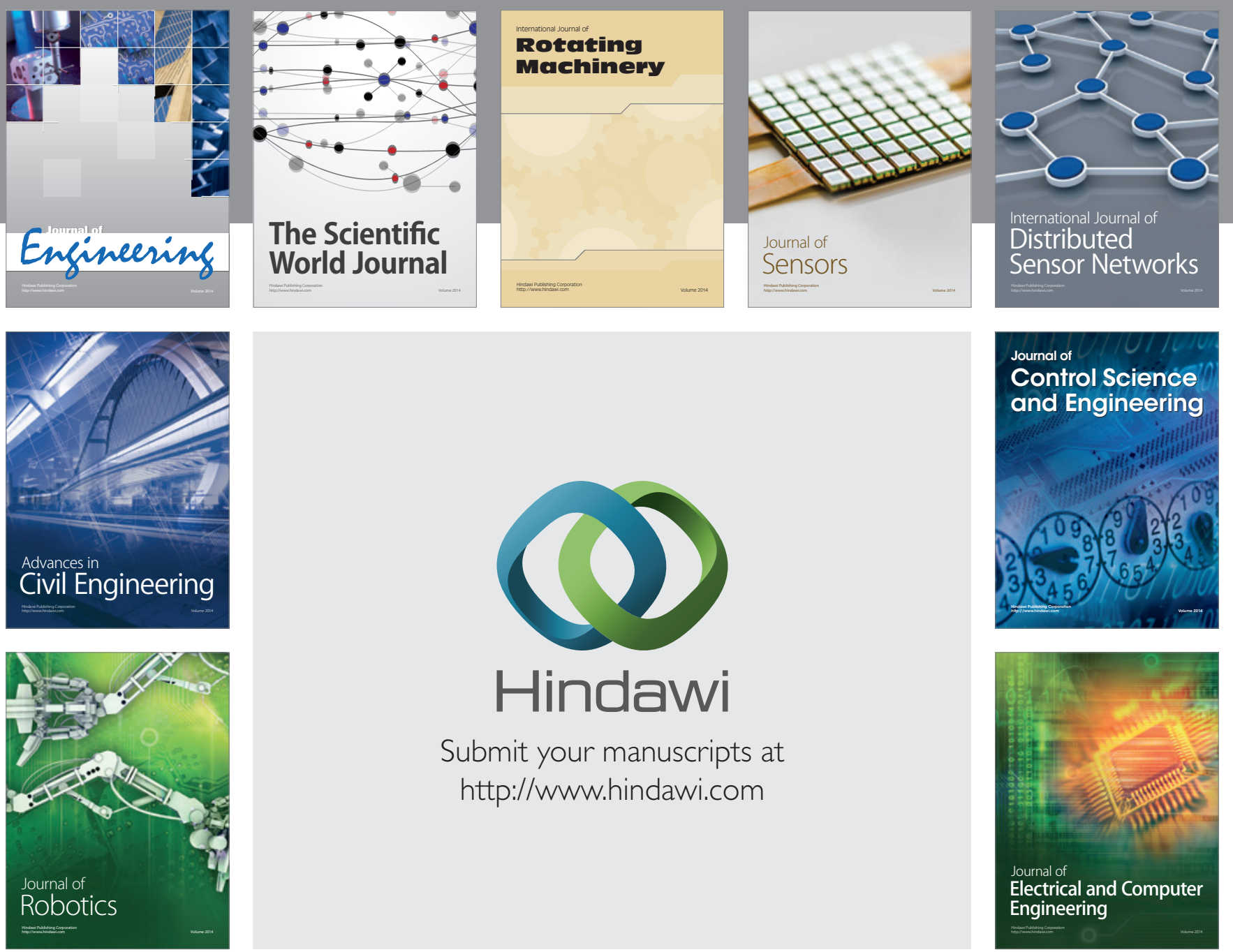

Submit your manuscripts at

http://www.hindawi.com
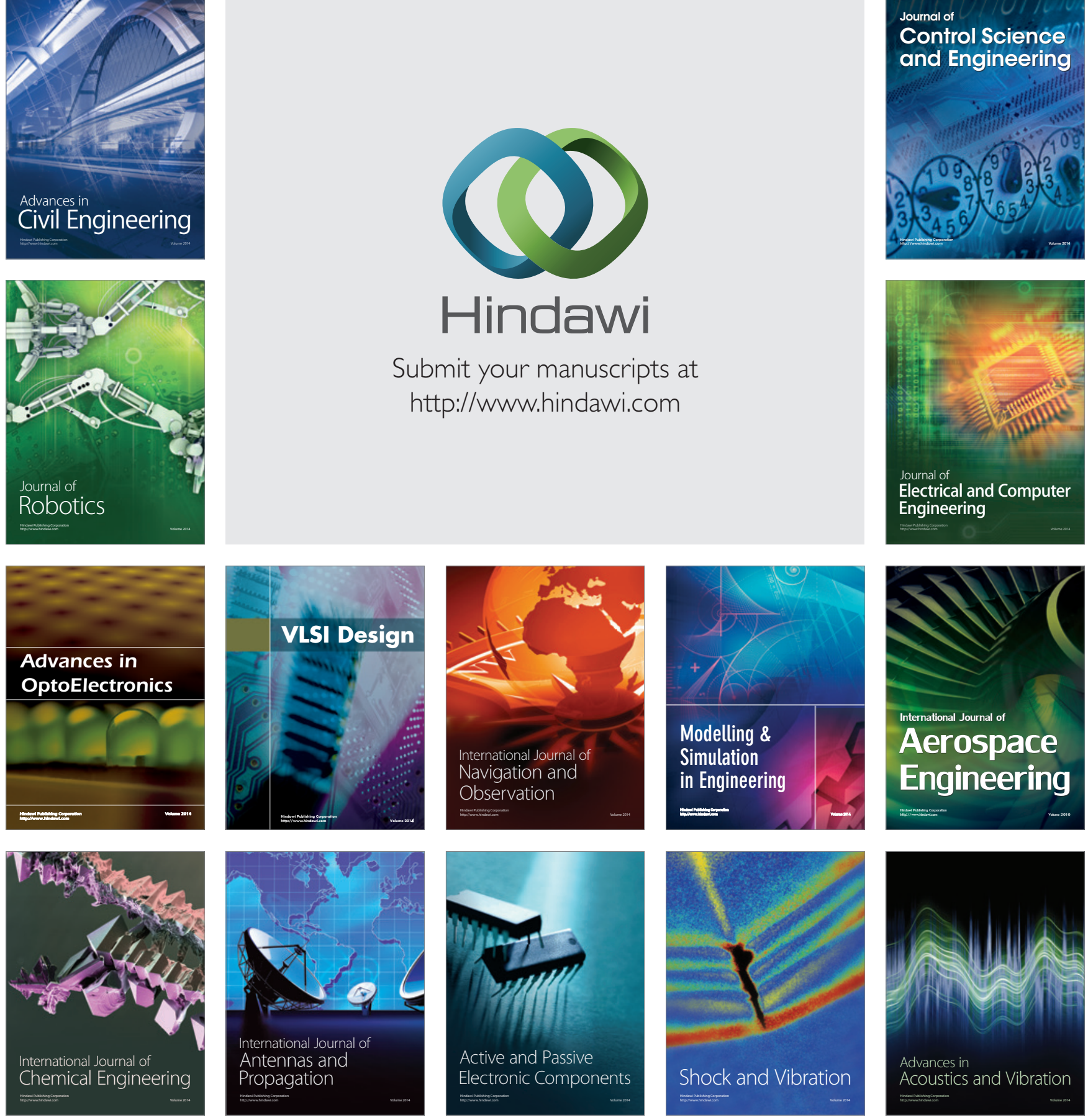